\title{
Re-présentations de la mort dans les médias d'information
}

Alain Rabatel et Marie-Laure Florea

\section{(2) OpenEdition}

1 Journals

Édition électronique

URL : http://journals.openedition.org/questionsdecommunication/401

DOI : 10.4000/questionsdecommunication.401

ISSN : 2259-8901

Éditeur

Presses universitaires de Lorraine

Édition imprimée

Date de publication : 30 juin 2011

Pagination : 7-28

ISBN : 978-2-8143-0084-2

ISSN : 1633-5961

\section{Référence électronique}

Alain Rabatel et Marie-Laure Florea, «Re-présentations de la mort dans les médias d'information », Questions de communication [En ligne], 19 | 2011, mis en ligne le 01 juillet 2012, consulté le 19 avril 2019. URL : http://journals.openedition.org/questionsdecommunication/401 ; DOI : 10.4000/ questionsdecommunication.401 
ALAIN RABATEL

Interactions, corpus, apprentissages, représentations, CNRS

Université Lumière-Lyon 2, ENs-Lyon

Alain.Rabatel@univ-lyon 1.fr

MARIE-LAURE FLOREA

Interactions, corpus, apprentissages, représentations, CNRS

Université Lumière-Lyon 2, ENs-Lyon

mlflorea@free.fr

\title{
RE-PRÉSENTATIONS DE LA MORT DANS LES MÉDIAS D'INFORMATION
}

«Montrez-moi la façon dont une Société s'occupe de ses morts, et je vous dirai avec une raisonnable exactitude les sentiments délicats de son peuple et sa fidélité envers un idéal élevé ».

Gladstone

\begin{abstract}
$\Lambda$
u fil des jours, les morts, la mort, figurent en bonne place dans les « pages internationales », à travers l'évocation des drames qui frappent des populations entières (catastrophes naturelles, famines, génocides, guerre, exactions des maffias ou des bandes armées mettant en coupe réglée des territoires entiers), dans les « pages nationales », à l'occasion du décès de personnalités de premier plan dans le monde des arts, de la politique notamment. La rubrique "société 》 (voire celle des « faits divers 》, sachant qu'en soi, l'appartenance à telle ou telle rubrique est riche de sens) fait écho à des décès emblématiques : suicides révélateurs des malaises d'une catégorie de la population (salariés de Renault, à Guyancourt, de France Télécom, policiers, détenus des prisons françaises); affaires liées à l'évolution des techniques ou des
\end{abstract}


mentalités, à l'instar des débats sur l'euthanasie, avec des cas fortement médiatisés (mort de Vincent Humbert en 2004, celle de Chantal Sébire en 2008, etc.). Même la rubrique sportive n'échappe pas à la mention sporadique de décès accidentels ou consécutifs à du dopage. Toutefois, malgré l'omniprésence de la mort dans les colonnes de la presse ou sur les écrans de télévision, le sujet reste très peu abordé par les analystes des médias, comme si le « tabou » qui entoure la mort se propageait jusqu'au domaine de la recherche. C'est cette lacune (relative certes, comme le montre l'état de l'art qui suivra, mais significative) que ce double dossier (le dossier de la 20 livraison portera sur «Évoquer la mort ») vise à combler, en portant un regard pluridisciplinaire sur les re-présentations contemporaines de la mort dans les médias d'information, les dossiers rassemblant des articles de sciences de l'information et de la communication, d'histoire, de sciences du langage, de sociologie. Après une mise au point sur la pertinence de la notion de re-présentation, on reviendra sur le paradoxe d'une mort à la fois omniprésente et absente dans les médias, souligné il y a quelques décennies déjà, avant d'évoquer certaines explications philosophiques, psychanalytiques, historiques et anthropologiques du phénomène - étant entendu que les 19 e et $20^{\mathrm{e}}$ livraisons tenteront de voir en quoi ce paradoxe s'est maintenu ou de mesurer ses évolutions. On s'intéressera ensuite à certains genres de discours sur la mort dans les médias avant de dégager quelques enjeux en lien avec les plans discursifs et sémiotiques et de présenter les contributions de ce dossier, centré autour de l'annonce de la mort.

\section{Les enjeux de la notion de re-présentation}

La notion de représentation est souvent critiquée en sciences de l'information et de la communication, en raison du peu d'intérêt heuristique qu'il y a à trouver dans les discours le reflet des événements (Krieg-Planque, 2006). Mais cette critique, bien que justifiée, n'épuise pas le débat. Le trait d'union avec lequel nous évoquons la re-présentation met l'accent sur le fait que cette notion est à envisager comme un procédé actif, puisque la mise en discours participe à la construction de l'événement, et non pas passif - car la représentation n'est pas simplement considérée comme une image plus ou moins fidèle de l'événement. Cette conception de la re-présentation insiste sur l'idée que la langue n'est pas le reflet d'un réel préexistant sur lequel elle n'aurait aucune prise. II est certain que des phénomènes physiques existent en dehors du langage, mais dans l'ordre des réalités sociales, les choses ne se produisent pas indépendamment des acteurs et donc du langage. En foi de quoi la linguistique (et l'analyse de discours, en particulier) ne doit pas chercher à retrouver dans les discours la confirmation de réalités ou de savoirs préexistants. En fait, une telle conception ne concerne pas que la linguistique : toutes les sciences qui utilisent des documents écrits ou oraux doivent dépasser les avatars du reflet ainsi que l'idée naïve selon laquelle la langue serait transparente à son objet comme aux intentions des acteurs qui 
agiraient dans la pleine conscience de ce qu'ils sont ou pensent (être). Or, si le rôle du langage dans les relations sociales comme dans la construction des objets (discursifs ou scientifiques) est une idée répandue, c'est souvent au prix d'une sous-estimation des spécificités langagières, comme en témoignent les propos de Bernard Lahire (1996: 124):

\begin{abstract}
« Une sociologie qui s'intéresse autant aux processus de construction des états structuraux ou aux systèmes, qui est autant préoccupée par la connaissance des modalités de l'incorporation du social que par les dispositions sociales déjà formées [...] est une sociologie nécessairement sensible aux pratiques langagières qui trament les formes de relations sociales. La pensée, les structures mentales ou cognitives ne s'inscrivent pas comme par miracle dans les cerveaux. Encouragé par les acquis de la psychologie vygotskienne, on peut donc souligner l'importance de la saisie des pratiques langagières à travers lesquelles prennent forme les rapports au monde et à autrui, eux-mêmes jamais dissociables des rapports de pouvoir et des rapports aux pouvoirs ».
\end{abstract}

Certes, Bernard Lahire a raison de ne pas séparer les pratiques langagières des autres pratiques sociales, raison aussi de considérer que les sciences sociales doivent prendre en compte le rôle du langage dans la construction des rapports interpersonnels, sociaux ou politiques comme dans celle des objets culturels et scientifiques. Néanmoins, si le linguiste se satisfait de la prise en compte du rôle social de la langue, il ne saurait cautionner toute dissolution de la langue dans le social - ou dans le psychologique, le cognitif, l'économique, etc. - : non seulement parce que la langue ne se réduit pas à la domination de facteurs externes (d'ordre social ou psychologique), mais encore en raison de la sous-estimation de l'opacité fondamentale de la langue, fortement mise en avant par Jacqueline Authier-Revuz (1995). Or, ce sont aussi ces non-coïncidences - entre les mots et les choses, entre ses mots et ceux des interlocuteurs ou de l'interdiscours - qui fondent la pertinence de la notion de re-présentation en tant que construction médiée et qui participent de la construction des pensées tout comme elles influent sur la compréhension des événements et, par là-même, sur leur cours. Or, on ne trouve guère d'échos de ce genre de préoccupations chez Bernard Lahire (1996). En d'autres termes, pas plus que la langue n'est le reflet d'actions préexistantes, elle n'est celui d'une lingua mentis toute élaborée - le mentalais des cognitivistes, tel que le théorise Jerry Fodor (1975). Au contraire, la langue construit les pensées en discours, elle les «présente » à travers de premières formulations et les « re-présente » à travers des processus de reprises, de reformulations, de paraphrases et d'ajustements du sens qui permettent d'envisager diverses facettes des objets discursifs, selon divers points de vue, cherchant à réduire les non-coïncidences sans jamais y parvenir. Mais il y a plus : les non-coïncidences ne sont pas seulement d'ordre langagier, elles relèvent encore de l'ordre politique, du vivre ensemble: les représentations, dont on sait qu'elles ne s'accordent pas toujours, posent la question de la recherche des meilleurs moyens de gérer les conflits, d'élaborer des modus vivendi. La dimension sociopolitique de la représentation est marquée par le lexique : « représentatif », « représentant » (le vendeur qui présente et vend les produits fabriqués par l'entreprise dont il est le vendeur patenté ou l'élu qui représente ses mandants devant la « représentation nationale », par exemple). Dans le monde complexe 
qui est le nôtre, il est important, nous dit Bruno Latour (2010 : 171-172), de trouver les meilleurs moyens de faire discuter ensemble des réseaux variables de sociabilités et de ne pas réserver les débats aux seuls initiés (responsables politiques ou économiques, savants), ni même aux experts (qui ne servent bien souvent qu'à légitimer les décisions sous le prétexte de la nécessité) en donnant au plus grand nombre possible de représentants, de porte-parole, la possibilité d'échanger. Bref, dans un monde vraiment cosmopolite,

« toute la question cosmopolitique se transforme alors en celle de trouver le moyen de construire les enceintes où ces porte-parole s'assembleront et partageront leurs incertitudes sur la qualité de leurs représentations. Ce mot vénérable doit être pris dans les deux sens, celui de la philosophie politique (qu'est-ce qu'un gouvernement représentatif?) et celui de la philosophie des sciences (qu'est-ce qu'une représentation exacte?) [...] Les représentants sont-ils légitimes et autorisés (qu'ils soient savants ou politiques?) Les représentations des choses et des affaires dont ils débattent sont-elles assez précises? Et, enfin, existe-t-il des enceintes légitimes pour qu'ils se retrouvent et puissent, éventuellement, changer d'avis à leur propos? » (ibid.).

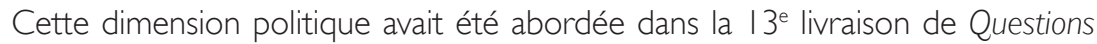
de communication (2008) à partir de la question de la responsabilité collective. Elle n'est pas centrale dans tous les articles des deux dossiers, mais elle émerge plus ou moins fortement lorsqu'il s'agit d'évoquer des conflits, y compris dans les re-présentations de la mort, dans la 20 livraison. II n'est pas sans intérêt de remarquer que la saisie du caractère construit des re-présentations facilite la remise en question du diktat de l'évidence qui accompagne les conceptions naïves de la représentation, au plan cognitif comme au plan politique, et, ce faisant, renvoie à de nouveaux débats, préparant de nouvelles décisions, de nouvelles actions, influant sur le cours des choses. Dans cette optique, au plan linguistique et, plus largement, sémiotique, la re-présentation, en tant que construction nouvelle, est une mise en scène jouant sur des effets de réel, à des fins argumentatives particulières, en sorte que la re-présentation est la trace de points de vue de l'énonciateur, quand bien même prend-elle l'apparence d'une re-présentation objectivante des choses. Et cette construction mérite d'être analysée, y compris lorsque les représentations tentent d'être le plus exactes possibles. De plus, la répétition de la re-présentation, sous une forme identique ou avec quelques variations, signale que la re-présentation est plus qu'une façon de donner du sens au vécu, elle en est une réexpérienciation. Or, cette dimension a fondamentalement à voir avec la fonction rituelle de l'évocation de la mort et du mort, pour faciliter le travail du deuil, comme on le verra plus loin.

\section{Morts partout, mort nulle part?}

Commençons par un bref aperçu de l'état de la question. Selon une formule de Louis-VincentThomas (2000 : 104), la mort dans les médias est partout et nulle part, « obscène et ab-scène ». Partout et nulle part, la formule mérite d'être précisée, car ce sont les morts qui sont partout, et c'est plutôt la mort qui est nulle part, 
« ab-scène » et ce sont, en effet, les images complaisantes et voyeuristes des morts qui sont obscènes, tout particulièrement dans les fictions, dont les médias font un large usage. Mais, dans les médias d'information, si les morts sont omniprésents (et donc « la mort », par métonymie), les discours sur la mort ou les morts, en tant que genres réglés, dans des rubriques dédiées, sont peu nombreux. II en va de même des photos d'agonie, ne serait-ce que faute de caméraman présent à ce moment-là Néanmoins, des évolutions sociétales et technologiques (les photos ou films pris à partir de téléphones portables par des particuliers) ont modifié quelque peu la donne. Cela dit, au-delà de la simple possibilité technique, la diffusion d'images d'agonie se heurte à des questions éthiques complexes : en dehors du fait que montrer l'agonie ou le corps d'une personne est moralement discutable, la mort « réelle » est propre à heurter la sensibilité des spectateurs, contrairement à la mort dans les fictions. Quoi qu'il en soit, ces images restent plutôt rares (ne serait-ce que par comparaison avec leur fréquence dans les fictions). Cette contradiction ne saurait étonner, moins en raison d'une insuffisance de réflexion philosophique sur la question qu'à cause de la terreur que l'expérience de sa propre mort est de nature à susciter. La présence de la mort ou des morts dans les médias dépend d'un certain nombre de facteurs : d'abord de la nature du média, télévision, radio, presse écrite, nouveaux médias en ligne. La périodicité entre également en ligne de compte: les médias soumis à une périodicité quotidienne ou hebdomadaire parlent rarement des événements heureux, ou des «trains qui arrivent à l'heure 》 et leurs contraintes éditoriales, centrées sur l'événement, voire le spectaculaire, pèsent sur la sélection et le traitement des informations. En revanche, dès qu'on sort de la fréquence quotidienne ou hebdomadaire, la tyrannie de l'actualité se fait plus légère, en sorte que la place des re-présentations des morts peut s'en trouver relativisée au profit d'une dimension plus réflexive sur la mort ou telle ou telle de ses manifestations. Cette relativisation dépend bien évidemment de la nature des supports, des lectorats et des choix de politique éditoriale. II n'y a donc pas un discours sur la mort homogène dans des médias qui seraient eux-mêmes homogènes. Ce constat dressé voilà près de vingt ans par Marc Lits (1993) est sans doute plus que jamais d'actualité aujourd'hui. Dans cet ouvrage, Frédéric Antoine (1993 : 50) soulignait la forte disparité entre l'omniprésence de la mort et des morts à la télévision et sa rareté en Une des journaux, non sans mettre l'accent sur de fortes disparités de traitement entre chaînes publiques et privées, entre la mort « sauvage » des faits divers violents avec ses gros plans et la mort « policée » des funérailles qui présente « un caractère plus rassurant ou en tout cas moins "choquant" pour le téléspectateur ». De fait, celui qui est pleuré, c'est l'individu tel qu'il était vivant, non le mort, ni la mort.

Au-delà de leur dimension factuelle, les recherches dirigées par Marc Lits (ibid. : 2 l) avaient le mérite de mettre en relief la complexité des motivations qui concouraient à la représentation médiatique de la mort:

« Outre la difficulté d'accepter l'idée d'une vision globale et généralisante des médias, alors qu'ils se distinguent assez sensiblement par leur traitement de la peur et de la violence, outre également une vision quelque peu périmée du rôle politique des médias comme régulateurs 
sociaux au seul service du pouvoir, il faut admettre que les médias jouent aussi avec notre propre désir de peur et de violence. La présentation systématique d'évènements ayant la violence et la mort comme dénominateur commun n'est pas simplement due aux obsessions nécrophiles de journalistes assoiffés de sang, elle n'est pas due non plus à un souci prophylactique des rédacteurs en chef soucieux du bien être de la population, elle répond certainement à des attentes inconscientes du lectorat ».

Cette complexité interdit de sombrer dans les facilités des incantations mettant en cause unilatéralement un public voyeur et avide de sensations fortes ou des médias sans scrupules lancés dans la seule course à l'audimat (ibid. : 26). Fondamentalement, il existe un paradoxe dont on voudrait cerner les tensions, aujourd'hui, entre une omniprésence de la mort et sa re-présentation « déréalisante » selon l'expression de Louis-VincentThomas ( 199 Ia :27), dans la mesure où la focalisation sur les morts accidentelles, violentes, est une façon somme toute commode d'envisager une mort « à laquelle on peut échapper et qui statistiquement reste de loin minoritaire ». Cette analyse vaut-elle aussi pour la re-présentation des grands drames humains et de leur cortège de cadavres? Les mises en scène de ces drames collectifs où le hasard et l'accident ont peu de place sont-elles tout aussi déréalisantes que les morts sauvages des faits divers? II semble qu'on puisse répondre par l'affirmative, si on les rapporte à l'expérience du vécu individuel de la mort. En ce sens, les mises en spectacle de la « mort anecdote » relèvent du divertissement pascalien (Thomas, 199la: 42-43; 199 Ib: 810-8II), avec son « bavardage rassurant » (Thomas, 1991a: 27), « comme si le discours télévisé sur la mort ne pouvait s'affirmer à l'écran qu'en décalage avec la réalité de l'expérience » :

« Médecine des temps post-modernes, l'image télévisuelle du mort revêt une fonction anxiolytique, le sacrifice quotidien d'un à deux êtres humains sur l'autel du journal télévisé permettant à chaque spectateur, dans le confort de son salon, d'accepter la mort en tant que phénomène général inévitable, tout en récusant la sienne » (Antoine, 1993 : 64).

L'objectif de ce dossier est donc, entre autres projets, de prendre la mesure des évolutions qui ont eu cours depuis ces analyses, alors que de profonds changements ont imprimé leurs marques, dans l'organisation de la vie sociale, culturelle, affective de nos sociétés, comme dans les modes de fonctionnement des médias. À l'aune de ces changements, y a-t-il des infléchissements comparables en matière de re-présentation de la mort et des morts? Si oui, dans quels domaines, selon quelles formes, et en quel(s) sens? Et de même, quelles significations accorder à l'absence éventuelle de changement(s)?

\section{L'arrière-plan des représentations de la mort dans les médias}

Dans cette perspective, il semble indispensable de revenir sur quelques travaux célèbres grâce auxquels les pratiques des médias méritent d'être interrogées. En effet, les re-présentations de la mort dans les médias sont indissociables de 
l'arrière-plan philosophique, anthropologique et historique dans lequel elles s'inscrivent.

\section{Arrière-plan philosophique}

Dans La mort (1977), Vladimir Jankélévitch distingue trois temporalités qui scandent un rapport évolutif à la mort. D'abord, « la mort en-deçà de la mort », celle que l'on médite, que l'on prépare, et qui n'est pas la mort; c'est une mort si abstraite qu'on peut penser pouvoir lui échapper, puisqu'on ne sait ni le lieu ni l'heure de sa mort. Les vieillards n'accèdent pas à cette connaissance : même si le terme se rapproche, il reste toujours incertain (mors certa, hora incerta). Au fond, il n'y a guère que le condamné à mort et le suicidé qui connaissent le terme. Au sein de cette première expérience de la mort en-deçà de la mort, existent trois scansions successives: la première la considère comme une éventualité abstraite (c'est l'état du mortalis), la deuxième envisage une mort probable qui se rapproche (moriturus), la troisième étant celle de la mort imminente (moribundus). Après « la mort en-deçà de la mort » vient « la mort dans l'instant mortel », phénomène irréversible, irrévocable, par lequel on meurt et on devient d'emblée absent à soi-même : «Ainsi donc le vivant vit sa mort dans un instant unique, et séance tenante il en meurt: car on ne peut vivre la mort sans aussitôt en mourir! On la vit en la mourant, et on meurt de la vivre » (Jankélévitch, 1977: 35I). C'est par abus de langage que la deuxième modalité du rapport à la mort est une expérience inscrite dans le temps, puisque sa survenue est le signe de notre disparition : « La mort ne s'apprend pas. II n'y a rien à apprendre en elle. D'abord c'est une chose qu'on ne fait qu'une fois dans sa vie, et cette première fois est aussi la dernière, par définition » (ibid. : 34). Enfin, « la mort au-delà de la mort » est celle qui est appréhendée sous une forme eschatologique, faisant place à la méditation sur l'au-delà, avec ses angoisses et ses espérances. Mais on quitte ici le terrain de l'expérience intime et physique de la mort pour une appréhension plus réflexive. Ces trois temporalités sont en relation avec trois modes d'appréhension distincts de la mort. II existe ainsi ce que Jankélévitch nomme avec bonheur « la mort en troisième personne » : c'est « la mort-en-général, la mort abstraite et anonyme, ou bien la mort propre, en tant que celle-ci est impersonnellement et conceptuellement envisagée » (ibid. : 25). Cette mort en troisième personne concerne bien sûr les autres, mais elle concerne aussi le sujet qui, pour paraphraser Jacques Madaule, sait qu'il va mourir, mais n'y croit pas. C'est pourquoi ce mode d'appréhension concerne à la fois « la mort avant la mort » et « la mort au-delà de la mort ». Le deuxième mode d'appréhension de la mort est « la mort en deuxième personne », c'està-dire celle du proche, qui est celui par lequel la mort distante nous frappe dans la sphère de l'altérité qui nous est la plus proche: « Le Toi représente en effet le premier Autre, l'autre immédiatement autre et le non moi en son point de tangence avec le moi, la limite prochaine de l'altérité » (ibid. : 29). Enfin, 
vient la mort en première personne, toujours vécue au futur, mystère qui reste inaccessible au sujet, car lorsque la mort dans l'instant mortel survient, le sujet trépasse: « Je suis toujours en effet, et par définition même, avant ma propre mort; le pendant, et a fortiori l'après, me sont obstinément refusés » (ibid. : 32). Cette mort en première personne est un mystère auquel il est impossible de se soustraire, tant il est vrai que malgré les tentatives de mises à distance, l'être humain pressent le «problème »: «Mea res agitur! C'est de moi qu'il s'agit » (ibid. : 26).

II vaut la peine de se demander à quelles temporalités et à quel mode d'envisagement de la mort les médias font généralement écho. Les médias d'information accordent une place massive à la mort en troisième personne. La mort en deuxième personne connaît un essor important : si jusqu'à présent, elle était cantonnée à certains genres dédiés (à l'instar des faire-part de décès ou des nécrologies), l'usage des nouvelles technologies et l'évolution des mœurs (qui permettent à tout un chacun de se faire journaliste et donc qui font des acteurs de l'événement les auteurs de l'information) ouvrent de nouvelles perspectives: ainsi une jeune mère a-t-elle mis en scène sur son blog la vie et la mort de sa fille Faith, atteinte d'anencéphalie (et donc vouée à une mort rapide)'. Quant à la mort en première personne, elle a peu de place dans les médias d'information, encore que là aussi, les nouveaux médias bouleversent la donne : ainsi de cette jeune actrice anglaise, Jade Goody, atteinte du cancer qui a fait entrer chez elle les caméras pour ses derniers mois de vie, afin de laisser de quoi vivre à ses enfants². Certes, la caméra n'a pas filmé ses derniers jours, mais cette éventualité se produira - si ce n'est déjà fait, dans des pratiques sous le manteau. De la même façon, de plus en plus de suicides sont, sinon filmés (ce qui arrive, mais c'est rapidement censuré), du moins annoncés en direct sur l'internet. Quoi qu'il en soit, c'est plutôt dans le domaine de la fiction que la mort en première et en deuxième personne se déploie (sans exclure la mort en troisième personne), tandis que la presse d'information fait des choix inverses. Cela étant, il convient de dépasser ce premier constat et c'est un des objectifs de ces numéros que de vérifier concrètement comment des évocations de la mort (en première, deuxième ou troisième personnes) traitent du sujet sur un mode distancié ou non, pathétique ou non, sensationnel ou non, d'analyser comment elles font place à l'émotion et à la réflexion, etc. De la même façon, il conviendra de voir dans quelles mesures les différents médias d'information font place aux différentes temporalités du rapport à la mort, et notamment si des évolutions ont pu être observées, plus particulièrement du côté des médias électroniques qui bouleversent les pratiques médiatiques.

\footnotetext{
' Le blog en question, qui date de 2009, se trouve à l'adresse suivante : babyfaithhope.blogspot.com.

${ }^{2}$ Voir www.paperblog.fr/ 737050 /jade-goody-est-morte-du-cancer. Voir également la mort annoncée de Farrah Fawcett sur paperblog.
} 


\title{
Arrière-plan psychanalytique
}

Nous ne développerons pas des travaux bien connus et aisément accessibles, nous nous bornerons ici à souligner les passerelles entre ces travaux et les autres paradigmes scientifiques évoqués dans cette section. Les pulsions de vie et de mort sont fortement intriquées, quoique selon des agencements variables. Éros et Thanatos font tous deux l'objet de tabous généralisés, sous une forme ou sous une autre. C'est ce que montrent les travaux qui rendent compte des évolutions historiques de longue durée. En effet, il semble que le tabou sur la mort soit plus fort que celui qui frappe la sexualité (voir Didier, 1988 : 127; Ariès, 1977 : 574). Notre époque, depuis plusieurs décennies, tient - en apparence du moins, mais cette apparence a du sens - un discours relativement libéré sur le sexe et sur la sexualité, alors que la mort est comme frappée d'interdit. Jean Baudrillard (1976: 279) met bien cette évolution en perspective lorsqu'il dit que

\begin{abstract}
« parler de mort fait rire, d'un rire crispé et obscène. Parler de sexe ne provoque même plus cette réaction : le sexe est légal, seule la mort est pornographique ${ }^{3}$ La société, "libérant" la sexualité, la remplace progressivement par la mort dans la fonction de rite secret et d'interdit fondamental. Dans une phase antérieure, religieuse, la mort est révélée, reconnue, c'est la sexualité qui est interdite. C'est l'inverse aujourd'hui ».
\end{abstract}

Ce renversement évoqué par Jean Baudrillard invite à regarder de plus près ce qui se passe au plan historique, même s'il a aussi des répercussions aux plans psychanalytique et politique, à travers le profilage d'un Éros unidimensionnel qui asservit les individus à une nouvelle forme de marchandisation, celle du désir, ou, à tout le moins, d'un désir marchandisé (ibid. : 280). Ainsi, dans les sociétés développées depuis le milieu du $X X^{\text {e }}$ siècle, la mort fait-elle l'objet d'une véritable « exclusion » (Ariès, 1977 : 573-574) qui concerne les cadavres mais aussi l'expression du deuil, de plus en plus restreinte au domaine privé, comme si la mort était contagieuse, comme si son évocation revenait à céder à une pulsion morbide. Et le lecteur ne sera pas surpris que des psychologues et des psychanalystes critiquent ces tendances et mettent en garde contre le fait que c'est le mécanisme de l'exclusion de la mort qui est en lui-même morbide, tant pour les individus que pour les sociétés. D'où les tentatives des dernières décennies, pour penser, sous une forme ou une autre, le retour du refoulé. C'est notamment le cas des travaux pionniers d'Élisabeth Kübler-Ross (1969) pour restituer au malade puis au mourant sa dignité, pour faire droit à la souffrance et à la mort - tel est le cadre dans lequel les travaux sur l'euthanasie sont à penser, aux antipodes des travaux sur l'eugénisme. Car la médicalisation de la mort n'a en rien aboli les craintes de la mort, et le malade hérissé de tubes et de sondes prend la forme moderne (et tarifée) de nos angoisses, aussi terrifiantes que l'étaient autrefois les danses macabres...

3 J. Baudrillard fait écho à l'article célèbre de G. Gorer (1955). 


\section{Arrière-plan historique}

Le scandale de la mort traverse aussi les représentations historicisées (et européano- ou occidentalo-centristes) de la mort. Dans L'Homme devant la mort, Philippe Ariès (1977 : 596-608) distingue quatre périodes historiques qui marquent des évolutions dans le rapport historicisé de l'homme à la mort selon quatre paramètres essentiels, la conscience de soi, la défense de la société contre la nature sauvage, la croyance dans la survie et dans l'existence du mal:

- Avant le Xle siècle, « la mort de tous » est « la mort apprivoisée » : c'est une mort publique, acceptée, maîtrisée. Elle correspond au modèle chrétien dans lequel les défunts sont familiers, où le trépas n'interrompt pas la continuité de l'être. Sur ce plan, la mort apprivoisée diffère de la mort familière de l'antiquité, qui craignait la mort et installait ses nécropoles en dehors des villes. II faudra attendre le $V^{e}$ siècle pour que les morts entrent dans les villes, dans et autour des églises, en lien avec le culte des martyrs et l'espérance dans la résurrection des morts (ibid. : 55).

- Du Xle à la fin du XVIle siècle, c'est « la mort de soi » : la mort se personnalise, la vie et la singularité de l'existence s'affirment, les sépultures s'individualisent, avec les chapelles des nobles avant de se « démocratiser » à travers l'usage des caveaux (ibid. : 287). Les cadavres sont de plus en plus occultés - le cercueil est très vite fermé, recouvert d'un poële, voire, pour les grands, d'une « représentation », nom donné à l'effigie funéraire en bois ou en cire du défunt (ibid. : I 68- 170). La pratique du testament se répand, en lien avec la montée en puissance de la famille nucléaire.

- Du XIXe au XXe siècle, « la mort de toi » correspond à la période romantique où la mort de l'aimé est vécue comme un arrachement théâtralisé, mais où se lit l'espérance des retrouvailles après la mort (ibid. : 604-605).

- Depuis environ le milieu du XXe siècle, avec « la mort inversée » ou « la mort interdite », la mort se médicalise, se professionnalise, avec des intervenants censés aider à une mort douce.

Toutefois, ces évolutions ne sont pas unilinéaires. Après le triomphe de la mort chrétienne médiévale, MichelVovelle (1974) souligne l'émergence d'une nouvelle sensibilité avec la Renaissance qui voit le recul des pestes et du macabre et connaît une triple contestation, religieuse (le protestantisme ampute l'au-delà du purgatoire), humaniste (le souci de la vie bonne au quotidien réduit la pensée de la mort) et populaire (l'enfer est tourné en dérision). De même, la période entre 1580 et 1730, qui correspond à la période baroque, est caractérisée par une « main basse sur la mort ». Au XIX siècle, la bourgeoisie « colonise la mort à son profit », tandis que le prêtre recule devant le médecin, que le deuil familial s'épanouit dans les cimetières. Alors, toutes les formes de croyance coexistent, certitude de la vie éternelle, incrédulité absolue, pensée religieuse, 
philosophique de la mort, espérance religieuse ou politique, etc. (Vovelle, 1974; 1983). II faut également garder en mémoire le fait que ces évolutions ont leur géographie : ainsi « la mort inversée », fortement développée auX USA et en Angleterre au $X X^{e}$ siècle, connaît-elle des résistances plus ou moins fortes sur le continent européen; de même, l'escamotage de la mort est plus fort dans l'Europe catholique ou protestante que dans l'Europe orthodoxe (voir Ariès, 1977 : 169-170; 587-588). De plus, la périodisation de Philippe Ariès ne recouvre pas les typologies philosophiques de Vladimir Jankélévitch - même si elle s'en inspire - mais elle a le mérite de rappeler que le terreau sur lequel elles ont émergé n'échappe pas à une longue durée qui, à la prendre en compte, relativise la portée des analyses par trop centrées sur une expérience européenne, et, qui plus est, sur une expérience très récente de quelques siècles seulement. En sorte qu'il y a(urait) du dommage à ne pas faire droit aux tendances lourdes de l'anthropologie, c'est-à-dire au fait que la mort est toujours « rôdeuse et refoulée, bien qu'omniprésente dans les médias, subite et souvent violente dans les guerres, les accidents, les catastrophes; ou lente s'il y a longue maladie, agonie ou coma prolongés; institutionnalisée et bureaucratique; de plus en plus médicalisée et mercantilisée » (Thomas, 199 lb: 822-823; Hintermeyer, 2003); de plus en plus individualisée (Elias, 1982). Autant dire que l'horizon anthropologique, sous-jacent dans les travaux historiques, s'avère une donnée d'arrière-plan fondamentale pour prendre la mesure des re-présentations de la mort dans les médias d'information, et qu'il doit être pris en compte, y compris pour mesurer la portée exacte des évolutions historiques des re-présentations de la mort.

\section{Arrière-plan anthropologique}

Si la mort est omniprésente dans les médias, il n'en reste pas moins que, dans nos sociétés développées, avec l'allongement de l'espérance de vie et le rétrécissement des familles à la famille nucléaire, les occasions personnelles de contact avec la mort sont rares. Par exemple, il a été calculé qu'aux USA on pouvait vivre 20 ans sans rencontrer un défunt dans son entourage. II n'en va bien sûr pas de même en Afrique ou en Inde. Bref, la disparition (relative) de la mort - qui peut s'accompagner de sa « démystérisation » et de sa désacralisation - ne l'empêche pas de rester un tabou ou un scandale (Thomas, I99 Ib : 836).

Encore convient-il de préciser que le rapport à la mort qu'on vient d'évoquer correspond aux conceptions des sociétés occidentales (sur lesquelles on se concentrera dans ce dossier eu égard à la perspective médiatique) et qu'il n'est pas partagé - loin de là - par d'autres cultures, comme le montrent notamment les travaux de Louis-VincentThomas sur l'Afrique.Au demeurant, dans les sociétés occidentales elles-mêmes, le rapport à la mort n'est pas homogène. Ainsi les travaux de Tony Walter (1994) ont-ils mis en avant un renouveau de pratiques mortuaires dans le domaine anglo-saxon, qui semble remettre en cause les 
analyses Philippe Ariès et de Louis-Vincent Thomas (notamment), dans la mesure où ce revival reposerait sur le refus du déni de la mort. En effet, la bonne mort qu'évoque Tony Walter, celle qui est choisie, préparée, maitrisée, qui est vécue par le mourant avec des proches (famille ou communauté d'appartenance) ou des accompagnants (professionnels de la mort) à son écoute, signifie-t-elle, autant que le dit l'auteur, la fin du déni de la mort? On est en droit de se demander si cette revendication de maîtrise du processus, jusqu'au bout, ne s'apparente pas à une forme suprême de déni, par l'affirmation illusoire d'une toute puissance de l'individu et du groupe qui l'entoure. Quant au fait que ce revival marquerait une volonté de rupture avec les rituels d'antan, là encore, rien n'est moins sûr, car l'expérience privée de cette mort néo-moderne touche un public relativement limité. Faute de recul suffisant, on ne peut assurer que ces pratiques remplacent les rituels, en général; certes, les rituels peuvent évoluer, mais la nécessité des rituels paraît une constante anthropologique des sociétés, humaines. D'ailleurs, Tony Walter lui-même s'interroge dans la conclusion de son ouvrage sur la portée de ces évolutions (voir aussi Déchaux, 1996). II n'en reste pas moins que, dans toutes les sociétés coexistent des représentations de la bonne et de la mauvaise mort : quelles que soient leurs différences, les sociétés africaines (plutôt animistes) et occidentales (plutôt chrétiennes) se retrouvent dans l'idée qu'une bonne mort intervient le plus tard possible, respecte l'intégrité de la personne, touche celui qui meurt entouré des siens, au pays, et dont les affaires sont en ordre (Thomas, 199 lb: 823-827). Et il n'est sans doute pas déraisonnable de lire la multiplication des soins consacrés à l'approche de la mort comme l'écho affaibli des angoisses qu'elle suscite, qui alimentent la persistance du tabou, ou l'illusion qu'on pourrait y échapper...

\section{Le discours sur la mort et les morts dans les médias}

Aujourd'hui, les genres qui traitent directement de la mort dans les médias sont davantage centrés sur l'annonce de la mort que sur son évocation, disséminée dans des rubriques qui ne lui sont pas dédiées ${ }^{4}$.

\section{Des genres anciens aux genres contemporains}

Parmi les genres discursifs (Adam, Herman, Lugrin, 2000) réservés à la mort ou aux personnes décédées dans les médias - et exception faite des genres littéraires (voir sur ce point Ernst, 1983; 1988) -, bien avant les nécrologies, viennent en premier lieu les déplorations, les éloges funèbres, puis les oraisons funèbres. II existe aussi les épitaphes (Ariès, 1977: 214-227; Simonin, 1988; Urbain, 1989), ou encore les testaments - ce genre devenant à certaines

4 Ce texte (ainsi que les articles du dossier) a été écrit avant la parution de l'ouvrage d'Harrusch (20 I0). On y reviendra dans la présentation de la livraison 20 de Questions de communication. 
époques un genre littéraire (voir Ariès, 1977: 188-197; Favre, 1988). Mais ces genres ne se retrouvent pas dans les médias, sauf pour des raisons extrinsèques à la problématique de la mort, par exemple la nécessité d'abonder les rubriques culture ou faits divers; mais d'une façon générale, ils relèvent aujourd'hui de la sphère privée. Éventuellement, leur reproduction passe par des supports très spécialisés, professionnels ou partisans, qui correspondent à une sphère privée élargie à une communauté. En effet, il est rare que de tels discours accèdent aux grands supports nationaux, sauf dans des espaces dédiés et à propos de situations très codifiées, à l'instar des discours prononcés lors de son installation à l'Académie française par le nouvel académicien, qui fait l'éloge du prédécesseur dont il occupe désormais le fauteuil, dans Le Monde, dans le supplément littéraire du Figaro. Bref, ces genres sont fortement contraints par des données contextuelles: importance de la sphère du privé au détriment des anciennes sociabilités, petit nombre de personnes susceptibles de tels usages, étroitesse et confidentialité du circuit de diffusion, etc.

\section{Le cas des nécrologies}

Dans la période contemporaine, le genre le plus abondant est celui de la nécrologie. Celle-ci a une histoire récente: en 1835, le Dictionnaire de l'Académie Française consacre pour la première fois un article aux nécrologies, les définissant comme « certains petits écrits consacrés à la mémoire des personnes considérables mortes depuis peu de temps ». En 2007, le Petit Robert considère qu'une nécrologie est une « notice biographique consacrée à une personne morte récemment 》. La comparaison de ces deux définitions témoigne de la stabilité de cette pratique discursive au cours de ses deux siècles d'existence. Étymologiquement, « nécrologie » signifie discours sur un mort: de fait, la nécrologie est l'héritière de la tradition multimillénaire de l'éloge funèbre, qui consiste à parler en termes élogieux d'une personne disparue, et qui, selon les lieux et les époques, se décline en elogium (dans l'Antiquité romaine), en oraison funèbre (en contexte religieux) ou en manifesto mortuario (ces affiches qui fleurissent dans les rues des pays méditerranéens lors d'un décès). La mort a ainsi toujours été l'occasion du surgissement de la parole, destinée à surmonter son chagrin, à entretenir la mémoire du disparu ou à rassembler ceux qui restent : en cela, la nécrologie fait partie des rites modernes du deuil occidental et de la mise en scène des valeurs essentielles d'une société (Dominicy, Frédéric, 200I). Tout au long du XIXe siècle. les journaux publient des nécrologies qui se présentent sous la forme de textes, écrits par les journalistes, qui retracent la biographie de personnes disparues d'une certaine notoriété. Mais ces textes mettront du temps à se faire leur propre place au sein du journal : leur parution est irrégulière, de même que leur place dans le journal, puisqu'ils paraissent sous des rubriques très diverses, des « Nouvelles » aux « Faits divers ». La fin du XIXe siècle voit l'apparition de véritables rubriques nécrologiques, qui occupent une place fixe dans le journal, et prennent la forme 
qu'on leur connaît aujourd'hui (Makarova, 2003). Depuis, la rubrique nécrologique s'est stabilisée et elle fait maintenant partie des pratiques bien ancrées dans l'usage journalistique. Aujourd'hui, le rubricage du journal fait une place régulière aux nécrologies qui bénéficient d'un espace dédié, généralement dans les pages du « carnet », et le nom de la rubrique, même s'il ne mentionne pas le terme « nécrologie », est souvent explicite. Ainsi les articles nécrologiques du Monde et du Figaro (les deux principaux pourvoyeurs de nécrologie parmi les titres de la presse nationale française) sont-ils respectivement surmontés de l'indication « Disparitions » et « Deuil ». À cet égard, on notera que le tabou qui entoure la mort fait obstacle à l'emploi du terme « nécrologie » dans les colonnes du journal : alors même que les articles nécrologiques parlent explicitement de la mort, pour les titres, des euphémismes sont souvent préférés aux termes plus crus. Le texte des nécrologies est lui-même aujourd'hui très codifié : après un court paragraphe destiné à annoncer le décès (et parfois les circonstances dans lesquelles il est survenu : la date, le lieu, la cause, etc.), l'article oscille habituellement entre récit de vie et hommage plus ou moins appuyé. La longueur de la nécrologie, quant à elle, est variable, pouvant aller de la simple brève à l'article d'une double page, selon la notoriété du disparu (Revaz, 2000; Florea, 2010).

\section{Les annonces nécrologiques}

À la différence des variations qui affectent le genre de la nécrologie, le genre des annonces nécrologiques paraît plus fortement contraint par ses dimensions et par le support médiatique. Ces faire-part de décès rédigés par les proches du disparu sont en effet des textes dont la diffusion est payante, qui obéissent à des routines assez fixes, partiellement déterminées par les journaux, qui imposent certaines normes de rédaction, mais aussi par l'usage, qui fixe certaines pratiques, notamment en ce qui concerne les formules utilisées ou la place des images dans les annonces (Hammer, 2010). Toutefois, ce genre présente néanmoins des spécificités et des sous-genres significatifs en matière de discours de re-présentation de la mort et du mort (Ringlet, 1993 : 73-74; Bertrand, 1995). Ce genre n'échappe pas aux évolutions sociétales qu'il traduit et accompagne (Ringlet, 1992, 1993), comme on le verra dans ce dossier.

\section{Quelques enjeux de la représentation discursive et pluri-sémiotique de la mort et des morts dans les médias}

\section{Des questions vives}

Mais la problématique des discours sur la mort/le(s) mort(s) ne saurait se limiter à l'étude, pourtant capitale, des nécrologies ou des annonces nécrologiques. Elle 
concerne aussi les discours qui, sans appartenir spécifiquement à un genre de discours sur la mort, au sens précédent, font néanmoins effort pour penser le fait de la mort, l'inscrire dans une chaîne évènementielle plus vaste. En ce sens, les discours médiatiques sont une façon d'investiguer un certain nombre de questions vives, ou, parfois, sont l'indice de la difficulté à les traiter frontalement. Ainsi des débats sur l'euthanasie, sur les rapports entre le suicide et le travail, sur tel ou tel génocide - génocide arménien, extermination des Juifs et des Tziganes sous le nazisme (Thanassekos, 2007) -, sur les famines ou sur tel ou tel conflit par exemple les conflits des Balkans où se mit en œuvre la purification ethnique (Krieg-Planque, 2003), le Rwanda (Halen, Walter, 2008; Fleury, Walter, 2008) ou encore des critiques artistiques qui rendent compte d'œuvres portant sur la mort. Dans tous les cas, ils interrogent la responsabilité journalistique, par ses choix comme par ses silences, et, au-delà, celle de l'ensemble du corps social et de ses corps intermédiaires (Rabatel, Chauvin, 2006; Rabatel, Koren, 2008, Rabatel, 2008). Ces questions-là sont le plus souvent abordées à travers l'évocation des enjeux de la mort.

\section{Ineffabilité?}

\| existe une « thèse » (sinon un poncif, à tout le moins, un raccourci fâcheux) selon laquelle la mort serait irreprésentable, indicible, et ne devrait son ineffabilité qu'au fait que la mort est un défi inconnaissable, générateur d'angoisses ou de tabous (Thomas, 2000: 105-106). Sans prétendre en rien nier la réalité de ces états de fait, notons toutefois qu'ils concernent plus la mort en première personne que les autres formes de rapport à la mort, sauf, bien sûr, à les rapporter à ses propres angoisses. C'est en fonction de cela que Louis-Vincent Thomas (ibid. : 60, 221) évoque diverses formes d'indicibilité : i liste ainsi les procédés lexicaux d'euphémisation, de tabouisation, de silence, ou encore les périphrases, circonlocutions. Celles-ci peuvent adopter soit un registre symbolique (《il s'est éteint »), soit vulgaire (《 il est/a crevé »), confirmant « le rôle évident des formules argotiques à fonction de catharsis » (Thomas, 199 |b : 805). Enfin, il rappelle que si vivre compte en français trois ou quatre synonymes, mourir en compte plus de trente, sans compter les périphrases (Thomas, 199 lb: 838). Au plan des escamotages ${ }^{5}$ discursifs qui peuvent reposer sur l'ellipse ou sur le « délayage par la rhétorique » (ibid. : 221-222), on a coutume d'accorder une grande importance aux récits, comme le fait Vladimir Jankélévitch, puisque l'en-deçà et l'au-delà échappant au discours, il ne resterait guère que « le récit de

\footnotetext{
${ }^{5}$ Les escamotages existant au plan personnel peuvent être les cadavres qui sont arrangés, parfois selon des techniques très sophistiquées (voir la thanatopraxie aux USA). Les vivants n'osent plus exprimer trop leur douleur; le port d'un brassard noir est tombé en désuétude, celui des vêtements du deuil l'est également. Pourtant, comme d'aucuns le reconnaissent, c'était à un signe tangible de peine qui était susceptible de provoquer les échanges, et d'aider avec douceur au travail du deuil (Châtelet, 2004).
} 
l'en-deçà, qui est biographie, et le roman de l'au-delà, qui est conte fantastique » (ibid.). Participent également de ces escamotages les inversions apophatiques qui tentent de dire la mort par ce qu'elle n'est pas - à supposer que la mort soit le contraire ou le contradictoire de la vie. Or, parler d'indicibilité est réducteur et opacifiant si l'on ne précise pas à quel ensemble de phénomènes on renvoie. Certes, il existe bien un phénomène mental, psychologique et cultu(r)el qui relève de la difficulté de dire, ou des interdictions de dire. Mais pour le linguiste, ce qui se dit est dit, et ce qui est dit est toujours significatif. Bien sûr, ce n'est pas la même chose de dire directement ou indirectement, selon tel ou tel registre; le mode de donation des référents, leur organisation dans la phrase et dans le discours sont significatifs. Au plan linguistique, donc, l'ineffabilité n'a aucune pertinence. II en va de même pour les re-présentations picturales de la mort ou des morts : même si elles contournent parfois la représentation crue de la mort, elles ne la montrent pas moins, avec des stratégies qu'il convient de décrire.

\section{Rôle du récit dans les représentations de la mort}

Qui plus est, il est réducteur d'interpréter le récit sous la seule fonction de l'escamotage par défaut ou par excès, réducteur également de laisser penser que le récit serait la forme unique ou privilégiée d'évocation de la mort. Précisons ces deux points. Certes, le récit entre dans la construction de l'identité des groupes sociaux. Certes encore, les médias ont pris le relais, sans les supplanter totalement, des mythes, des contes et légendes ou des textes littéraires. La prégnance des re-présentations de la mort/des morts (dans la presse écrite ou télévisée) dit le monde, institue notre vision du réel en nous le racontant. Tout cela est si fort que, par une généralisation abusive, on en vient parfois à étendre les contours de l'identité narrative, qui est bien réelle dans sa sphère, en considérant que l'identité se construit obligatoirement à travers les récits, renommés « grands récits » pour l'occasion. À cet égard, la mode du storytelling (Salmon, 2007) dans les médias nous semble correspondre à une simplification (médiatique, mais théorique aussi), car le récit ne saurait être l'alpha et l'oméga de la construction des identités. II faut donc rappeler que l'identité déborde la question de l'identité narrative (Ricœur, 1983; Charaudeau, 1997). Comme le souligne Jean-Marc Ferry (1991 : 1 |2), à côté du rôle fondateur de la narration, qui est le mode premier de la construction de l'identité, entrent en jeu d'autres registres. Ainsi l'interprétation intervient-t-elle « dès lors que le récit est compris dans une perspective d'édification », quand la réflexion dépasse le cadre contingent de l'action pour expliquer ce qui est. Ainsi encore de ce que l'auteur entend en un troisième temps, l'argumentation, qu'il définit comme un effort pour penser les phénomènes particuliers et leur explication selon des logiques différentes, orientées vers des raisons d'agir valables pour tous, et pas seulement pour ceux qui ont été les auteurs ou les témoins de tel évènement qui s'est trouvé raconté puis interprété. C'est pourquoi l'argumentation est elle-même appréhendée à 
partir des nécessités d'un quatrième mouvement qui parachève la dynamique de construction des identités, que Jean-Marc Ferry nomme la « reconstruction », qui vise à justifier l'argumentation en prenant en compte d'autres bonnes raisons, en les situant, en les contextualisant, afin d'éviter des dérives idéologiques intolérantes, sans pour autant verser dans le relativisme. C'est bien l'ensemble de ces niveaux qui interagissent dans la construction émotionnelle et rationnelle des identités, centrée sur l'action, elle-même chargée d'émotion et de raison. La dynamique des catégories élaborées par Jean-Marc Ferry est précieuse pour penser la multiplicité des biais par lesquels les médias d'information annoncent et évoquent la mort, et, surtout, l'intrication des phénomènes langagiers et cognitifs autour de ces re-présentations qui savent jouer sur les émotions et l'empathie, mais s'efforcent aussi de penser rationnellement les enjeux de la mort et de ses re-présentations. Ainsi les nécrologies, les articles consacrés à tel génocide ou à tel problème de société ne font-ils pas que raconter des événements dramatiques, ils les mettent en perspective, les analysent, voire tentent d'envisager de possibles solutions; certes pas à la mort en tant que processus inéluctable, mais à la mort en tant que scandale révélateur de drames dont il devrait être pensable ou souhaitable de faire l'économie. On voit qu'à la lumière des analyses précédentes, l'évocation de la mort ou des morts dans les récits est plus complexe que ce qu'en disent Vladimir Jankélévitch et LouisVincent Thomas et que, au demeurant, le récit gagne à être articulé avec d'autres modes de représentation, à visée argumentative et interprétative, qui jouent leur rôle dans l'appréhension philosophique et anthropologique de la mort, des morts.

\section{Annoncer la mort}

Les textes composant les $19^{e}$ et $20^{e}$ livraisons de Questions de communication traiteront la problématique de la re-présentation de la mort en s'attachant, pour la livraison 19, aux modalités de la re-présentation de la mort dans les médias d'information (Annoncer la mort), et, pour la livraison 20, aux enjeux de la re-présentation de la mort dans les médias d'information (Évoquer la mort). Bien sûr, les deux dossiers traitent ensemble, quoique selon des proportions différentes, des formes de la re-présentation et de leurs enjeux. « Annoncer » la mort, c'est annoncer le plus souvent une mort individuelle plutôt que des morts collectives ou des morts en masse. C'est aussi célébrer le mort - et, comme on le verra, célébrer le vivant qu'il a été, la vie qui continue à travers des valeurs partagées. Autrement dit, annoncer la mort fait partie des rituels de la mort, et c'est à partir de cette notion de rituel que l'on s'efforcera de penser sa re-présentation discursive et (télé)visuelle. En revanche, évoquer la mort, c'est traiter le plus souvent de la mort comme signe de, des morts accusatrices, des morts qui interrogent, bref, des morts-parce que, des mortspour éviter que. La dimension évocative se fait analytique et praxique, renvoyant 
à des drames politiques, à des crises sociales auxquelles il s'agit de trouver des solutions pour réduire le nombre de décès. Certes, cette dimension se retrouve dans l'annonce de la mort, mais l'évocation y est davantage chargée d'émotion, dans la mesure où elle est souvent assurée par des locuteurs qui entretiennent une certaine proximité avec le disparu et où elle est contrainte par des dispositifs génériques.

Ce dossier cherche à déterminer comment le mort (ou la mort, voire le vivant?) est dit(e) et montré(e) dans les médias d'information. Sa subdivision prend en compte les types de médias afin de vérifier si la mort est dite à l'identique à la télévision ou dans la presse, si les nouveaux médias en ligne ont fait émerger de nouvelles façons de parler de la mort, plus personnelles, plus immédiates. Surtout, le présent numéro entend interroger ces modes de re-présentation afin de voir s'ils sont une façon de reconduire, sinon les formes des rituels, du moins les fonctions immémoriales des rituels, par lesquelles les mots, comme les gestes sans oublier qu'en l'occurrence les mots sont des gestes discursifs - témoignent de l'engagement de ceux qui font cortège au mort, afin de mettre des mots sur des émotions, de permettre la séparation d'avec le mort et le travail du deuil. Bref, il s'agit de voir sous quelles formes les vivants font mémoire de celui qui part, tout en faisant société pour faciliter le retour à la vie qui doit continuer.

Le dossier se répartit en trois sous-ensembles : le premier est consacré à l'analyse des nécrologies et les faire-part de décès dans la presse écrite de la dernière décennie. Le deuxième s'intéresse à de nouveaux rituels commémoratifs sur la toile. Le troisième traite des évolutions des funérailles télévisées en France depuis l'origine. Marie-Laure Florea montre que dans les nécrologies, la re-présentation de la mort fait l'objet d'une scénographie qui marque la rupture entre vivants et défunt, dans le même temps que le mort est re-présenté, au sens où il est rendu à nouveau présent par le biais du discours. De la sorte, la nécrologie remplit sa fonction rituelle, dans la mesure où l'évocation du disparu permet non seulement le travail de deuil, mais aussi de faire société autour des valeurs que le mort incarnait au plus haut point, et qui s'avèrent indispensables à la permanence de la société ou de communautés plus restreintes. Françoise Hammer met en évidence des stratégies de contournement du tabou de la mort dans les fairepart de décès publiés dans la presse régionale. Par-delà la fonction pragmatique première d'annonce du décès et de la date des obsèques, elle dégage notamment quatre stratégies discursives de distanciation, de convocation, d'invocation et d'appropriation de la mort.

Les deux articles suivants analysent de nouvelles pratiques d'écriture, qui sont autant de nouveaux modes de sociabilité autour des faire-part et des éloges mortuaires sur la toile. Adeline Wrona s'intéresse au site « jesuismort.com », qui renouvelle les formes de l'éloge post mortem, en hybridant des modèles hérités avec les potentialités propres aux écrits d'écrans. Le discours tenu sur les morts détermine des gestes communicationnels (offerts par le site) qui donnent sens à l'hommage et constituent autant de participations d'un nouveau genre à un rituel funéraire examiné du point 
de vue de son régime temporel, de son modèle économique, enfin de ses modes de sociabilité. Sophie Pène étudie trois phénomènes liés au fonctionnement du réseau social qu'est Facebook, les faire-part, les échanges consécutifs au maintien de la page de quelqu'un qui est décédé, ainsi que les commémorations de décès liés à des drames (catastrophes, crimes, maladies, etc.) : les mémoriaux y montrent les enjeux affectifs et symboliques qui traversent ces lieux de socialisation numérique, tout en s'interrogeant sur leur devenir.

Benoit Lafon analyse dans la longue durée la dimension cérémonielle des funérailles télévisées, depuis les origines de la télévision en France. L'expérience collective des obsèques en direct a varié dans sa façon de figurer les morts et d'impliquer les vivants, en faisant évoluer les représentations vers des hommages civils et des figures tutélaires renouvelées. Ces évolutions, à propos de figures qui ne sont pas des proches, témoignent de nouvelles modalités de neutralisation de la mort, fondées sur l'intersubjectivité, se substituant aux comportements traditionnels. Ce faisant, les funérailles télévisées sont révélatrices de la perpétuation sous des formes sans cesse renouvelées de «formalisations 》 et « reformalisations 》 (Elias, 1982) d'un rapport à la mort, par l'intermédiaire de la production de figures tutélaires.

\section{Références}

Adam J.-M., Herman T., Lugrin G., dirs., 2000, « Genres de la presse écrite et analyse de discours 》, Semen, 13.

Antoine F., 1993, « Mourir au JT. Les cadavres exquis de l'information télévisée », pp. 45-64, in : Lits M., dir., La peur, la mort et les médias, Bruxelles, Éd. Vie ouvrière.

Ariès Ph., 1975, Essai sur l'histoire de la mort en Occident du Moyen Âge à nos jours, Paris, Éd. Le Seuil.

— 1977, L'Homme devant la mort, Paris, Éd. Le Seuil.

Authier-Revuz J., 1995, Ces mots qui ne vont pas de soi, Paris, Larousse.

Baudrillard J., 1976, L'échange symbolique et la mort, Paris, Gallimard.

Bertrand Y., 1995, « Les faire-part de décès dans la presse allemande », Nouveaux cahiers d'allemand, vol. I3-4, pp. 389-399.

Charaudeau P., 1997, Le discours d'information médiatique. La construction du miroir social, Paris, Nathan.

Châtelet N., 2004, La dernière leçon. Paris, Éd. Le Seuil.

Danblon E., 200I, « La rationalité du discours épidictique », pp. 19-47, in : Dominicy M., Frédéric M., dirs, La mise en scène des valeurs. La rhétorique de l'éloge et du blâme, Paris, Delachaux et Niestlé.

Déchaux J.-H., 1996, «Walter (Tony). The revival of death », Revue française de sociologie, 37, pp. $16 \mid-164$. 
Delporte C., dir., 2003, « Interdits. Tabous, transgressions, censures », Le temps des médias, I.

Didier B., 1988, « Le journal intime : écriture de la mort ou vie de l'écriture », pp. 127- | 47, in : Ernst G., dir., La mort dans le texte, Lyon, Presses universitaires de Lyon.

Di Folco P., dir., 20 I0, Dictionnaire de la Mort, Paris, Larousse.

Dominicy M., Frédéric M., dirs., 200 I, La mise en scène des valeurs. La rhétorique de l'éloge et du blâme, Paris, Delachaux et Niestlé.

Dubied A., Lits M., 1999, Le fait divers, Paris, Presses universitaires de France.

Elias N., 1982, La solitude des mourants, trad. de l'allemand par S. Muller Paris, Christian Bourgois, 1998.

Ernst G., dir., 1983, La mort en toutes lettres, Nancy, Presses universitaires de Nancy.

1988, La mort dans le texte, Lyon, Presses universitaires de Lyon.

Favre M., 1998, « La mort-caution : l'écriture testamentaire », pp. I03-I I 0, in : Ernst G., dir., La mort dans le texte, Lyon, Presses universitaires de Lyon.

Ferry J.-M., 1991, Les puissances de l'expérience. Essai sur l'identité contemporaine, Paris, Éd. Le Cerf.

Fleury B., Walter J., dirs., 2008, Qualifier, disqualifier, requalifier des lieux de détention, de concentration et d'extermination, Nancy, Presses universitaires de Nancy.

Florea M.-L., 2010a, « Nécrologies », pp. 735-737, in : Di Folco P., dir., Dictionnaire de la Mort, Paris, Larousse.

— 20 I Ob, « Interpeller l'absent : le rôle de la convocation du disparu dans les nécrologies », in : Torterat F., Thibaut, A., dirs., Corela, L'interpellation. Accès : http ://corela.edel.univpoitiers.fr/index.php?id=743.

- 2010c, « Portrait d'autrui et image de soi : l'éthos dans la nécrologie de presse », pp. 63-72, in : Actes du XXVe Congrès international de linguistique et de philologie romanes, T.V, Berlin, De Gruyter.

Fodor J., 1975, The Language of Thought, New York, Crowell.

Freud S., 1931, L'inquiétante étrangeté et autres essais, trad. de l'allemand par B. Féron Paris, Gallimard.

Gorer G., 1955, « Pornography of Death », Death, Grief and Mourning in Contemporary Britain, London, Cresset Press, pp. 169- 175.

Halbur B.,Vandagriff M., 1987, « Societal responses after death : a study of sex differences in Newspaper death notices for Birmingham, Alabama, 1900-1985 », Sex Roles, 17/7-8, pp. $421-436$.

Halen P., Walter J., dirs, 2008, Les langages de la mémoire. Littérature, médias, et génocide au Rwanda, Metz, Publications de l'université Paul Verlaine.

Hammer F., 2010, « Interpellativité et appellativité. Une étude pragmatique de l'avis de décès », in :Torterat F., Thibaut, A., dirs, Corela, L'interpellation. Accès : http ://corela.edel. univ-poitiers.fr/index.php?id=754.

Haneman B., 200 I, « On the writing and reading of obituaries », Medical journal of Australia, I7-I, pp. 59-60. 
Hanusch F., 20 I 0, Representing Death in the News. Journalism, Media and Mortality, Australia, Palgrave Macmillan.

Herman T., 200 I, « "Le Président est mort. Vive le Président". Images de soi dans l'éloge funèbre de François Mitterrand par Jacques Chirac », pp. 197-202, in : Dominicy M., Frédéric M., dirs, La mise en scène des valeurs. La rhétorique de l'éloge et du blâme, Paris, Delachaux et Niestlé.

Hintermeyer P., 2003, Euthanasie, la dignité en question, Paris, Buchet-Chastel.

Jacquin G., dir., 2003, Le récit de la mort. Écriture et histoire, Rennes, Presses universitaires de Rennes.

Jankélévitch V., 1977, La Mort, Paris, Flammarion

— 1994, Penser la mort, Paris, L. Levi.

Kresta R., 1996, " "Nachrufe" in englischen und deutschen Fachzeitschriften der Soziologie: Untersuchungen zu einer vernachlässigten Textsorte », Fachsprache, vol. I, 3-4, pp. I | 8-137.

Krieg-Planque A., 2003, «Purification ethnique ». Une formule et son histoire, Paris, CNRS Éd.

— 2006, «"Formules" et "lieux discursifs": propositions pour l'analyse du discours politique », Semen, 21. Accès : http ://semen.revues.org./I 938

Kübler-Ross E., 1969, Les derniers instants de la vie, trad. de l'anglais par C. Jubert et É. de Peyer, Genève, Labor et Fides, 1997.

Lahire B., 1996, « Inégalités, partages, spécificités et différences dans les usages sociaux de l'écrit », pp. I 15-128, in : Barré de Miniac C., dir., Vers une didactique de l'écriture. Pour une approche pluridisciplinaire, Bruxelles/Paris, DeBoeck/ INRP.

Latour B., 2010, Cogitamus. Six lettres sur les humanités scientifiques, Paris, Éd. La Découverte.

Lits M., dir., 1993, La peur, la mort et les médias, Bruxelles, Éd. Vie ouvrière.

— 1994, Le roi est mort. Émotions et médias, Bruxelles, Éd. Vie ouvrière.

— 1996, Récit, médias et société, Louvain-la-Neuve, Éd. Academia-Bruylant.

Long G., 1987, « Organizations and identity: Obituaries | 856-1972 », Social forces, 65-4, pp. 964-1001.

Makarova A., 2003, « Dits et non-dits des nécrologies de la presse », Le temps des médias, I, pp. 108-1 18.

Marion Ph., 1993, « Fictions de la peur. Peur immédiate et peur médiate », pp. 161 - 177, in : Lits M., dir, La peur, la mort et les médias, Bruxelles, Éd. Vie ouvrière.

Moore S., 2002, « Disinterring ideology from a corpus of obituaries: a critical post mortem », Discourse and society, 13-4, pp. 495-536.

Rabatel A., 2008, « Pour une conception éthique des débats politiques dans les médias: répondre de, devant, pour, ou les défis de la responsabilité collective », Questions de communication, I3, pp. 47-69.

- 2009, «L'arrestation de Jésus et la représentation de Judas en Jean, 18, 1-12. Une relecture mise en perspective avec l'univers de la gnose et l'Évangile de Judas », Études théologiques et religieuses, T. 84, vol. I, pp. 49-79. 
— 2010, « Le traitement médiatique des suicides à France Télécom de mai-juin à mi-août 2009 : la lente émergence de la responsabilité du management dans les suicides en lien avec le travail », Studia universitas Babes-Bolyai, Philologia, T. LV, vol. I, pp. 3 I -52.

Rabatel A., Chauvin-Vileno A., éds, 2006, « Effacement énonciatif et responsabilité dans les médias 》, Semen, 22.

— 2006, « La question de la responsabilité dans les médias », Semen, 22, pp. 5-24.

Rabatel A., Koren R., éds, 2008, « La responsabilité collective dans la presse », Questions de communication, 13, pp. 7-24.

Revaz F., 1997, « Le récit dans la presse écrite », Pratiques, 94, pp. 19-33.

— 2000, « La nécrologie : un genre rédactionnel? », Semen, 13, pp. 187-204.

Ringlet G., 1992, Ces chers disparus: essai sur les annonces nécrologiques dans la presse francophone, Paris, A. Michel.

1993, « Et à l'heure de notre mort. La peur en nécrologie », pp. 65-76, in : Lits M., dir., La peur, la mort et les médias, Bruxelles, Éd.Vie ouvrière.

Rivet D., 1994, « L'expression du fait religieux dans le carnet mortuaire du Temps et du Monde : de la fin du XIXe siècle à aujourd'hui », Chrétiens et sociétés $X V^{e}-X X^{e}$ siècles, I, pp. 7-34.

Roth K., Roth J., 1988, « Öffentliche Todesanzeigen (Flugblatt-Nekrologe) in Südosteuropa. Ein Beitrag zum Verhältnis zu Tod und Trauer », Österreichische Zeitschrift für Volkskunde, 3, pp. 253-267.

Salmon C., 2007, Storytelling. La machine à fabriquer des histoires et à formater les esprits, Paris, Éd. La Découverte.

Simonin M., 1988, « Ronsard et la tradition de l'épitaphios », pp. 85-99, in : Ernst G., dir., La mort dans le texte, Lyon, Presses universitaires de Lyon.

Thanassekos Y., 2007, « La rhétorique de la catastrophe », Questions de communication, I2, pp. $4 \mid-56$.

Thomas L.-V., I99 Ia, La mort en question, Paris, Éd. L'Harmattan.

— 199 lb, « L'homme et la mort », pp. 803-868, in : Poirier J., dir., Histoire des mœurs, T. 2 , Modes et modèles, Paris, Gallimard.

— 2000, Les chairs de la mort, Paris, Institut d'édition Sanofi-Synthélabo.

Urbain J.-D., 1989, L'archipel des morts. Le sentiment de la mort et les dérives de la mémoire dans les cimetières d'Occident, Paris, Plon.

Vernant J.-P., 1996, L'individu, la mort, l'amour. Soi-même et l'autre en Grèce ancienne, Paris, Gallimard.

Vovelle M., 1974, Mourir autrefois. Attitudes collectives devant la mort aux XVII et XVIIle siècles, Paris, Julliard.

— 1983, La mort et l'Occident de 1300 à nos jours, Paris, Gallimard.

Walter T., 1994, The Revival of Death, London, Routledge.

Walter J., 2005, La Shoah à l'épreuve de l'image, Paris, Presses universitaires de France. 